\title{
Quality of Service and Power Consumption Optimization on the IEEE 802.15.4 Pulse Sensor Node based on Internet of Things
}

\author{
Puput Dani Prasetyo Adi ${ }^{1}$, Akio Kitagawa ${ }^{2}$ \\ Micro Electronics Research Laboratory (MeRL) \\ Kanazawa University, Kanazawa, Ishikawa, Japan
}

\begin{abstract}
The Purpose of this research is to determine the Quality of Service (QoS) Zigbee or IEEE 802.15.4 sensor Node use the indicators, i.e. the Receiver Signal Strength and PathLoss (attenuation (-dB)) at the time of communication of the sensor node end device to the sensor Router node or Coordinator sensor node (sink). The factor power consumption sensor node is important to maintain the lifetime sensor node, The Sensor data in this research is the pulse sensor. The development of the Wireless Sensor Network communication system is in multi-hop communication, with efforts to obtain low power consumption on each sensor node. This study utilizes the Routing Protocol for Low Power and Lossy Network (RPL) method with position management on the sensor node on DODAGs consequently, that the average power consumption value for each sensor node is low. The benefit of the Sensor node is to send pulse sensor data from various nodes that are interconnected at different distances in multi-hop so that power consumption and Quality of Services (QoS) can be identified from the sensor node. From the research results, the average PathLoss value of IEEE 802.15.4 or Zigbee in free space is obtained by comparing the various simulation values and field experiments at a distance of $50 \mathrm{~m}$ at $-75.4 \mathrm{~dB}$ and the Average Receiver Signal Strength (RSS) with a comparison of Equation and Experiments in the field with parameters The minimum Power Transmitter is $0 \mathrm{dBm}$ and the Power Transmitter is maximum $+20 \mathrm{dBm}$ at a distance of $50 \mathrm{~m}$ at $-\mathbf{6 6 . 6}$ $\mathrm{dBm}$. Therefore, Pulse Sensor data will be displayed on the Web Page and stored in the MySQL database using Raspberry PI 3 as the Internet Gateway.
\end{abstract}

Keywords-RPL; RSS; Pathloss; Zigbee; Pulse; DODAGs; IoT

\section{INTRODUCTION}

Heart rate is an important thing that can be one of the first indicators for patients as a doctor uses a stethoscope. The normal range for Pulse sensor between 60-100 beat per minute (bpm), there are two conditions for abnormalities in the heart i.e. Bradycardia and Tachycardia. If the heart conditions are abnormal, consequently, the blood flow will also be disrupted, this disorder is Arrhythmia, i.e. the heartbeat conditions are too slow heartbeat (Bradycardia), too fast heartbeat (Tachycardia) or irregular or variable conditions. The parameters that can be calculated are signal strength (RSSI) and Weakening signal (PathLoss) using ZigBee.

In this research, node sensors are sent using Pulse sensors through the Free Space area, there are multi-hop sensors that are interconnected and therefore, the closest sensors will send pulse data to the edge node, and edge nodes then send pulse sensor data to Raspberry $\mathrm{Pi} 3$ as an internet gateway.

ZigBee or IEEE 802.15.4 Protocol technology was developed for the medical field, Zigbee specification is a light and comfortable prototype for patients, i.e. a pulse detection system using pulse sensors, the ability coordinator node to collect pulse sensors simultaneously without being discovered 3 (tree) nodes [1]. Zigbee has a frequency of $2.4 \mathrm{GHz}$ and a data rate of $250 \mathrm{kbps}$, this is low bandwidth and is right for the Wireless Sensor Network, Zigbee has the ability to transmit data up to 100 meters.

Perception Layer is a ZigBee Communication System that is used i.e. Star, Tree, and Mesh Topology Fig. 4, from this communication system traffic data, can be analyzed from End Device Node, Router Node and Coordinator Node, this data traffic includes Quality Of Services (QoS) i.e. Throughput, Packet Loss and Delay, and Radio Frequency (RF) capabilities in sending data indicated 21 y Radio Signals Strength (RSS) or Radio Signals Strength Indicator (RSSI) [2, 3, 4, 5, 6]. at previous research testing the performance of Raspberry Pi 3 in capturing radio waves with the Master-Slave system using an RF Bluetooth RN-42 device in sending multi LM35 sensor data, accordingly in this research study the RSSI value is different from the environment can be monitored, from this study obtained the value of precise RSSI [7, 8, 9, 10], furthermore, the next research will be developed of sensor node using a multi-sensor (pulse and blood Pressure sensor). In addition to Zigbee, the wider Wireless Sensor Network device is GSM SIM900A [11] with Ultrasonic sensor data which is often referred to as WMAN.

Furthermore, the development of Wireless Sensor Network technology is applied to UnderWater, which is called the Underwater Wireless Sensor Network (UWSN). In research [12] the analysis was carried out by measuring RF strength with Path Loss Parameters, Velocity of Propagation, absorption loss and the rate of signal loss in a different underwater environment.

The Wireless Sensor Network on this research must be robust and have low power consumption for each node, because the Wireless Sensor Networks to be built are very numerous and interconnected, in this case, at the free Space. Therefore, routing techniques are needed on the Wireless Sensor Network architecture. Routing Protocol for Low Power and Lossy Network is routing that is used on IEEE 802.15.4 or 
Zigbee protocol devices, Zigbee in this research is used on sensor nodes which will then function as senders of pulse sensor data. The pulse sensor type used is as in Fig. 1. Therefore, in this research there are two stages of research, 1) examine data transmission on sensor nodes with multi-hop networks. 2) Sending data to the coordinator node to the server at a certain distance with Pathloss and RSSI parameters in free space. The development of this research is towards an Internet of Things, because it is expected that this pulse sensor data can be an indicator for patients who want to check heart rate comfortably without having to go to the hospital, [7] in previous research the authors developed IoT using multisensor LM35 and Raspberry Pi 3.

Path Loss (PL) [13, 14, 15] is a weakening of Radio Frequency (RF) wave signals (in this research using ZigBee), which is caused by obstacles during the sending process from transmitter antenna to the receiving antenna, PathLoss (PL) $[16,17]$ is one of the indicators used to determine whether signal strength is good $(\mathrm{dB})[18,19]$ the external environment is influenced by Free-Space Path Loss (FSPL). Furthermore, Free-Space Path Loss (FSPL) [20, 21] measured using the Received Signal Strength Indicator (RSSI) technique using a module Zigbee. accordingly, there are two methods used to represent the results of PathLoss, i.e. in the condition of $n=2$ or free space, PathLoss is called FSPL (Free Space PathLoss) in outdoor conditions (no space) or no interference, such as other radio waves, in conditions, when the signal is emitted with a known variable from the antenna height which has an impact on reflecting the ground or reflected waves, and the second method is sending data from the edge node to Internet Gateway and pulse data storage in MySQL Database using Python language and HTML and JASON to display graphs on web pages and about IoT Security has not been discussed in this research.

\section{RELATED STUDIES}

Muhammad Niswar, Amil Ahmad Ilham, Elyas Palantei, Rhiza S. Sadjad, Andani Ahmad, Ansar Suyuti, Indra Bayu, Zaenab Muslimin, Tadjuddin Waris, and Puput Dani Prasetyo Adi [1], in their research entitled performance evaluation of ZigBee-based wireless sensor networks for patients' monitoring pulse status, this research is included in the Wireless Body Area Network (WBAN) other than Bluetooth, furthermore, from this research the performance of Zigbee as a sender of pulse sensor data is multiplexed or using the star network topology which causes a bottleneck in the coordinator node that functions as the receiver or antenna receiver. Finally, from the 5 sensor nodes that are sent together to the Coordinator node, they cause a loss when sending sensor data with 4 sensor nodes, so a maximum of only 3 sensor nodes or ZED can send data in full or maximum to the Coordinator node with a less than 30 distance meter.

Hana Mujlid, Ivica Kostanic [14], in their research entitled Propagation Path Loss Measurements for Wireless Sensor Networks in Sand and Dust Storms, in this study using empirical measurement data that is using Radio Frequency (RF) Zigbee or IEEE 802.15.4 which works on the $2.4 \mathrm{GHz}$ frequency. The research taken is measuring Path Loss in conditions of sandstorms and severe sandstorms that have an impact on the loss of propagation pathway from radio signals. From this research, the intercept values were 53 and $60 \mathrm{~dB}$ and the slope values were 28 and $37 \mathrm{~dB} /$ Dec.

Zhenran Gao, Weijing Li, Yan Zhu, Yongchao Tian, Fangrong Pang, Weixing Cao, and Jun $\mathrm{Ni}$ [22], in their research entitled Wireless Channel Propagation Characteristics and Modeling of Research in Rice Field Sensor Networks, in this study testing of Wireless Sensor Network devices in the location of agriculture or agriculture, is precisely in the rice field. So the result of sending signals from the Transmitter antenna to the Receiver antenna is affected by the growth of the rice field. testing was carried out at different altitudes $(0.8 \mathrm{~m}, 1.2 \mathrm{~m}, 1.6 \mathrm{~m}$, and $2.0 \mathrm{~m})$ consequently, that analysis of the shipment would be found data with different node heights, the models used are free space models and twoRay models.

Jose Vera-Pérez, David Todolí-Ferrandis, Salvador Santonja-Climent, Javier Silvestre-Blanes, and Víctor Sempere-Payá [23], The Routing Protocol for Low Power and Lossy Network (RPL) is considered for Low energy consumption in the Wireless Sensor Network i.e. IEEE 802.15.4 Network, to support the Internet of Things architecture. The parameters used in determining the quality of the Wireless sensor network i.e. Energy Consumption, Transmission Power, transmission receiver and Packet Size.

\section{METHODOLOGY}

\section{A. Pulse Sensor}

The pulse sensor used to detect Heart rate, there are 3 colors of the pin cable, e.g. the red cable for $\mathrm{VCC}$ equal to 3.3 - 5 Volts DC and Working Current is $4 \mathrm{~mA}$, black cable for GND and purple color cable for Signal, if the Green LED in the middle of the circle pulse the sensor lights up indicates the Pulse Sensor is ON or active. Accordingly, the Pulse signal sensor is entered on the Analog A0 Pin Arduino pro mini. In this research, Waveform Heartbeat can be shown using an Integrated Processing Development of Environment (IDE) that is integrated with Arduino Pro mini-IDE. This electronic pulse sensor uses a green LED that continuously emits light to the skin. As long as the heart beats, consequently, blood waves flow into the skin capillaries so that light absorption occurs extra during that period. And the process of absorption of light is considered to represent beats heart. Fig. 1 shows three views of a pulse sensor, which is front, back and enlarged, therefore, this is an important part of the pulse sensor that needs to be highlighted in this research [24].

\section{B. Heart Beat Data Heart Beat Data}

Pulse Wave or Photoplethysmogram (PPG) from Pulse sensor is shown in Fig. 2. It seems that a person's heart rate is $80 \mathrm{BPM}$, which means that the heartbeat of a normal person is a range of $60-100$ BPM $[1,24,25]$. Point $\mathrm{T}$ is the starting point of forming a PPG signal, and point $\mathrm{P}$ is the peak point of the pulse wave amplitude. The percentage value of $25 \%$ and $50 \%$ shows the magnitude of the Amplitude value generated on the pulse sensor. 


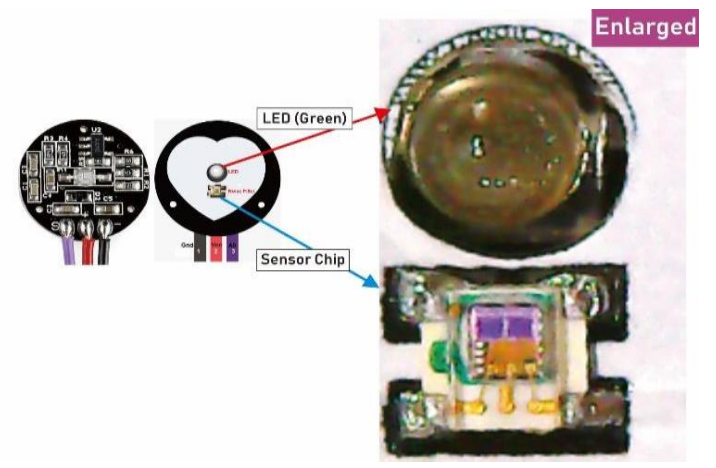

Fig. 1. Pulse Sensor

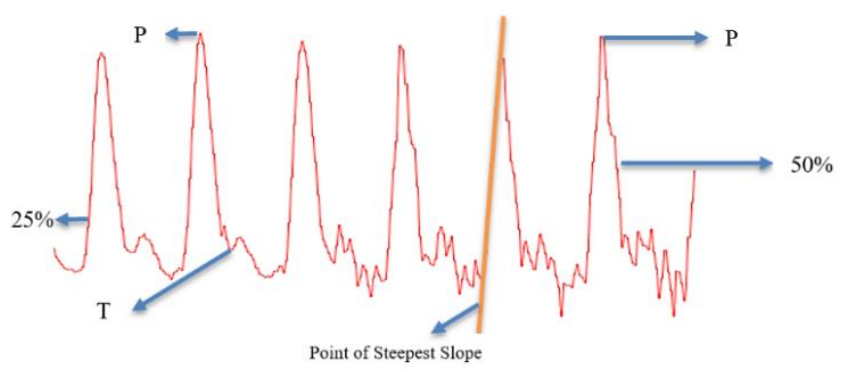

Fig. 2. Pulse Sensor Visualizer.

\section{Heartbeat or Pulse}

Arrhythmia is an abnormal condition in the heart, which is a slow pulse (Bradycardia), too fast (Tachycardia) or an everchanging or irregular pulse. Accordingly, the heart rate is 60100 BPM (beats per minute) [1]. Bradycardia is a condition of the heart rate below 60 BPM or below Normal, consequently, the condition causes a person to experience fatigue, weakness, dizziness, sweating, and fainting [1, 24, 25]. Conversely, Tachycardia is a condition of the heartbeat that is above 100 BPM or above normal, the condition of the heartbeat is too fast. Consequently, someone who has Tachycardia is dizzy, heart palpitations are very fast, fainting, mild headaches, shortness of breath, fatigue, in extreme situations sufferers can experience unconsciousness and Cardiac arrest. Moreover, the classification of heartbeat conditions is shown in Table I. therefore, this research Bradycardia and Tachycardia are important elements used as indicators on prototypes, where if BPM conditions show certain values such as Bradycardia then the LED indicator will show Yellow, conversely, if Tachycardia will show a red LED.

This pulse sensor data will be sent by the sensor node in the form of packet data (bytes) that can be monitored using XCTU software. The different distance comparisons will be used as parameters for determining Quality of Service (QoS) from the sensor nodes in this research. The QoS analyzed include Throughput (Kbps), Packet Loss (Packet), Receive Signal Strength (RSSI) and Path Loss (dB) as a result of transmitting sensors data. Accordingly, the test area is Free Space value of Path Loss exponent $(n)=2$.

\section{Zigbee $S 2 c$}

Zigbee works on the $2.4 \mathrm{GHz}$ frequency and includes the IEEE 802.15.4 protocol, Zigbee is an RF device that uses a low voltage of 2.1-3.6 Volt, generally on the ZigBee module is 3.3 volts, with Low current RX consumption is $18.8 \mathrm{~mA}$ and Tx is $17.4 \mathrm{~mA}, 9 \mathrm{~dB}$ of ZigBee gain and Average RF Power Transmitter $(\mathrm{Pt})$ Zigbee is $14.77 \mathrm{dBm}$, with a data rate of $250 \mathrm{kbps}$. Zigbee has many types, e.g. Zigbee S1 and ZigBee S2, ZigBee S1 are compatible RF devices with a point to point and point to multipoint, commonly referred to as star networking, while ZigBee S2 (Fig. 3) is an RF device compatible with mesh networking, usually used as a router. On a mesh networking, ZigBee has the ability to communicate between routers. In this research, ZigBee S2c is compatible with the Mesh Network. ZigBee is a Radio Device that emits radio waves in all directions where there is a request from another radio in a collection of sensor nodes so that the ZigBee antenna belongs to the isotropic type. Furthermore, ZigBee is also included in the Ad-Hoc Networking category because ZigBee is a collection of mobile Nodes in accordance with the ZigBee capacity is compatible with the Mesh Network or only a tree or star network. Fig. 5 Clustering method is used to group nodes based on the closest distance and form clusters and lifts 1 cluster head $(\mathrm{CH})$, therefore, Cluster Head is called Sink which is tasked with sending data of all node members to 1 cluster to Edge Router.

\section{E. Routing Protocol for Low Power and Lossy Network}

Routing Protocol for Low Power and Lossy Network (RPL) is a type of routing protocol based on the Destination Oriented Acyclic Graph (DODAG). DODAG is a special kind of DAG where each node wants to reach a single destination. The advantages of the Routing Protocol for Low Power and Lossy Network (RPL) are each embedded device or sensor node i.e. IEEE 802.15.4 or Low-Power Wi-Fi in large quantities can be connected effectively and optimally seen from limited power, memory, and processing resources. Accordingly, Fig. 6(a) and (b) are examples of DODAGs built using the Contiki cooja simulator.

The simulation in this research consists of Layer, Protocol and standards that are used to build simulation nodes, in detail can be seen in Table II [26]. The essence of RPL is an attempt to manage the energy or lifetime of a node.

TABLE I. INDICATOR ClASSIFICATION ON THE PROTOTYPE

\begin{tabular}{|l|l|l|}
\hline beats per minute $($ bpm $)$ & Classification & LED Indicator \\
\hline$>100$ & Tachycardia & Red \\
\hline 60 to 100 & Normal & Green \\
\hline$<60$ & Bradycardia & Yellow \\
\hline
\end{tabular}

TABLE II. PROTOCOL STACK COMMUNICATION

\begin{tabular}{|l|l|l|}
\hline Layer & Protocol & Standard \\
\hline Application & CoAP & IETF RFC 7252 \\
\hline Transport & UDP & IETF RFC 768 \\
\hline Network & IPv6 / RPL & IETF RFC 6550 \\
\hline Adaptation & 6lowpan & IETF RFC 6282 \\
\hline Data Link & IEEE 802.15.4 MAC (CSMA) & IEEE 802.15.4 \\
\hline Radio Duty Cycling & ContikiMAC & - \\
\hline Physical & IEEE 802.15.4 PHY & IEEE 802.15.4 \\
\hline
\end{tabular}




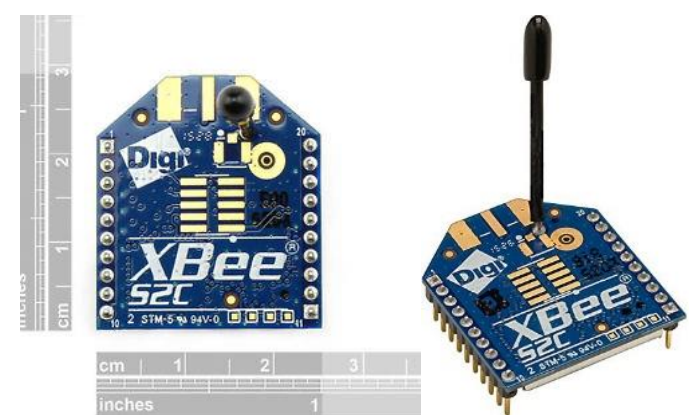

Fig. 3. Zigbee S2c.

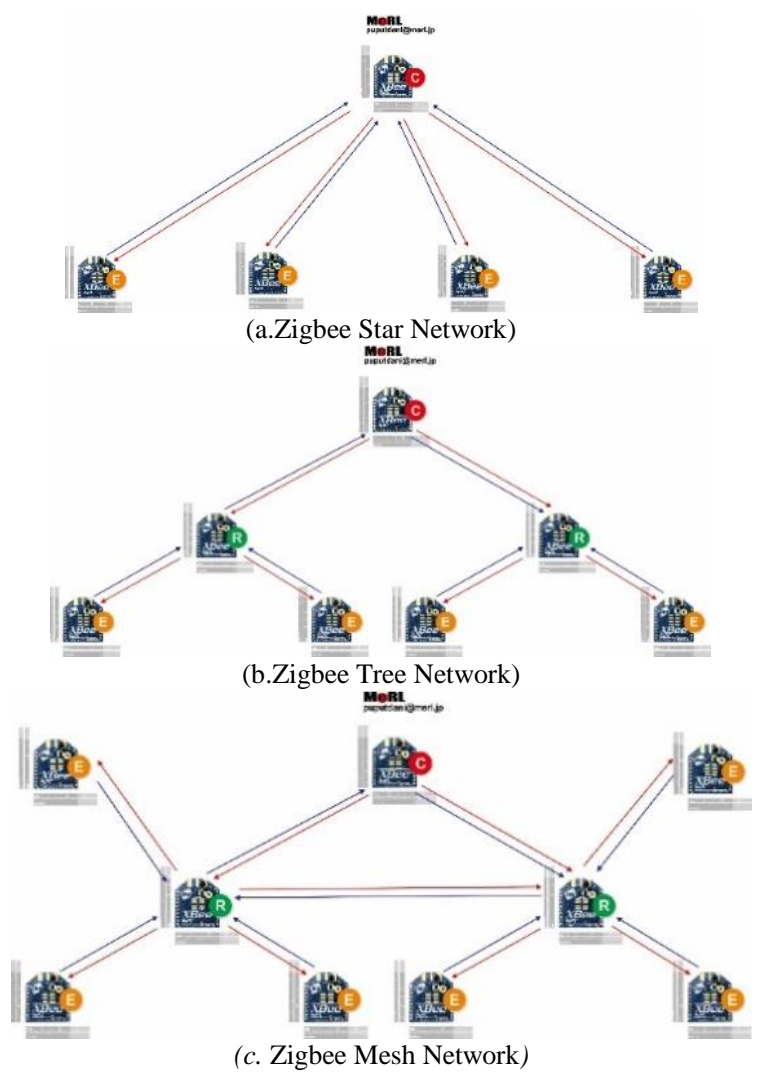

Fig. 4. Zigbee Topology (Star, Tree Dan Mesh Network).

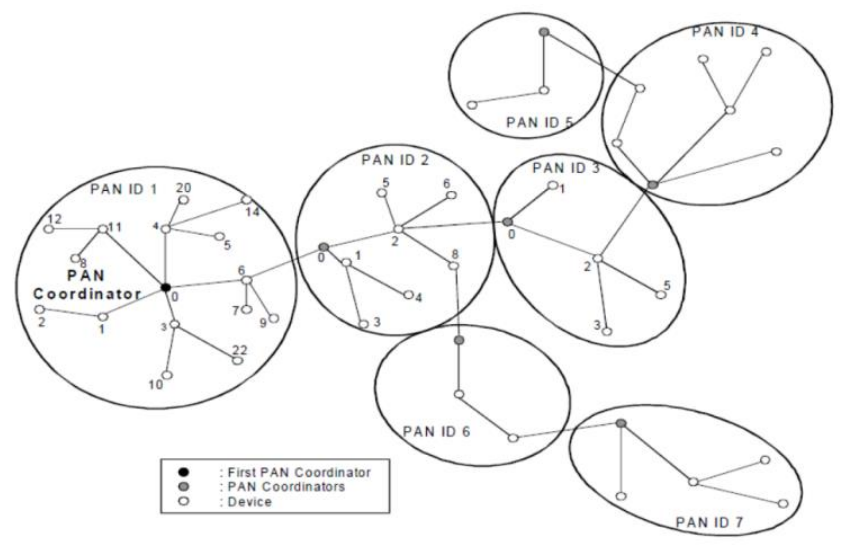

Fig. 5. Clustering LowPAN Network.

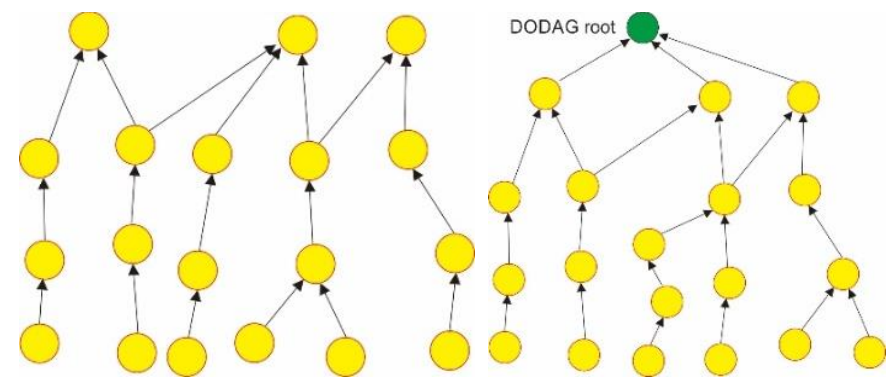

Fig. 6. (a)DAG (Directed Acyclic Graph) (b) Destination Oriented DAGs

\section{F. Pulse Sensor Node on IoT Architecture}

The architecture that will be built is shown in Fig. 7. There are two main analyzes, i.e. transmit sensor nodes on multihops which are indicated by setting the router node to EDGE router or sink. The first analyzes are the Quality of Services (QoS) this is an area to calculate the path loss and Receiver Signal Strength parameters. Furthermore, the next stage after the data arrives at the EDGE Router, the analysis is carried out on the Internet Gateway, setting up the MySQL database and display on the WEB so that any devices can see in real-time the Pulse Data sensor [27]. At this step is a need of an analysis of Reability and Energy Consumption in IoT.

\section{G. Hardware}

Zigbee Sensor nodes in this research shown in the following Fig. 8, built from a pulse, this sensor has been built from Arduino pro mini microcontroller and XBee S2c, this sensor node is ZED (ZigBee End Devices). In this node sensor prototype use $8 \times 2$ I2C LCDs and 3 LED colors with each 220 ohm resistor, the indicator espouse to read the patient's pulse condition. Furthermore, Logic Level Converter (LLC) is used as a regulator, LLC consists of several Pin High voltage i.e. 5 Volt and Low Voltage 3.3 Volt Pin. accordingly, which requires a 3.3 volt voltage is an $8 \times 2 \mathrm{I} 2 \mathrm{C}$ and XBee S2c LCD, the pulse sensor can use 3.3 volts or 5 volts, furthermore, the battery used as a power supply is a 3.7 volt $1000 \mathrm{mAh}$ battery type, while the FTDI 232 is used as an Arduino Pro mini board programmer from Arduino Integrated Development Environment (IDE) that uses $\mathrm{C}++$ programming language, the library such as library pulse sensor, LCD and interrupt to calibrate the results of Pulse sensors, Precise material selection will make it convenient to use nodes for patients. Table III shows the hardware functionality of the sensor node.

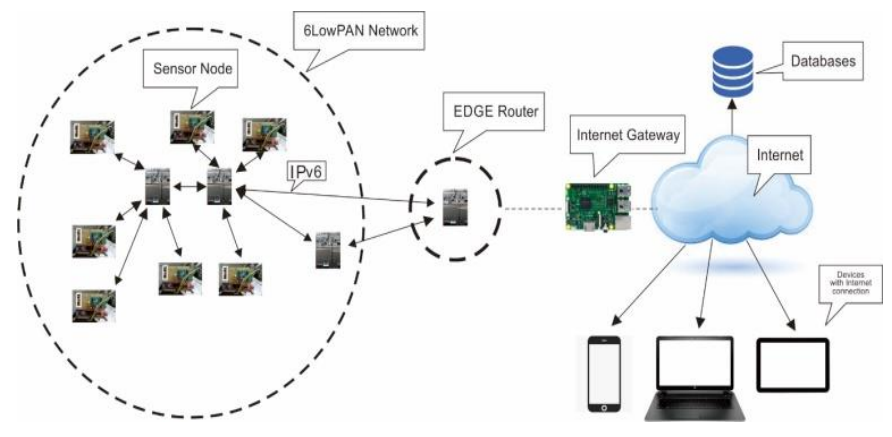

Fig. 7. Pulse Sensor Node on IoT Architecture. 


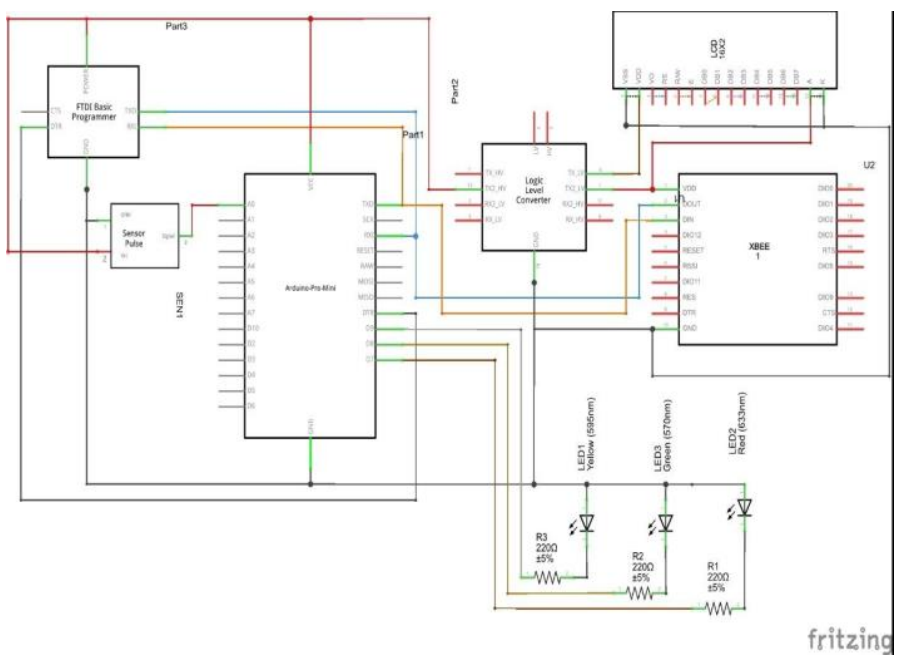

Fig. 8. Node Sensor Schematic.

Table III shows the hardware and working functions on the sensor node, there are three types of ZigBee settings, e.g. Zigbee coordinator, Zigbee router and Zigbee end devices each have different functions, voltage usage is 3.3 volts and 5 volts on a sensor node, therefore, the voltage will be distributed, this is where the regulator works by using the High Voltage (HV) to Low Voltage (LV) and Low Voltage (LV) to High Voltage (HV). On Arduino pro mini bootloader programming is needed unlike the Arduino Uno version, so FTDI 232 is needed as a bootloader.

TABLE III. NODE HARDWARE

\begin{tabular}{|c|c|c|}
\hline No & Hardware & Notes \\
\hline 1 & $\begin{array}{l}\text { Microcontroller } \\
\text { Arduino Pro mini }\end{array}$ & $\begin{array}{l}\text { Processor, ADC, Data Serial Communication } \\
\text { With a Base Station }\end{array}$ \\
\hline 2 & $\begin{array}{l}\text { XBee S2c End } \\
\text { Device }\end{array}$ & $\begin{array}{l}\text { Wireless sensor Network type to sending Pulse } \\
\text { sensor data to Coordinator node }\end{array}$ \\
\hline 3 & $\begin{array}{l}\text { XBee S2c } \\
\text { Coordinator }\end{array}$ & $\begin{array}{l}\text { Wireless sensor Network type to receive Pulse } \\
\text { sensor data from ZED or ZR to Base Station }\end{array}$ \\
\hline 4 & XBee S2c Router & $\begin{array}{l}\text { Wireless sensor Network type to sending Pulse } \\
\text { sensor data to Coordinator node from ZED and } \\
\text { Communicate between each Router at Mesh } \\
\text { network }\end{array}$ \\
\hline 5 & $\begin{array}{l}\text { XBee USB } \\
\text { Adapter }\end{array}$ & Setting Device to configuration the ZigBee S2c \\
\hline 6 & Pulse Sensor & As Pulse Sensor data from patients \\
\hline 7 & $\begin{array}{l}\text { LCD } 16 X 2 \text { atau } \\
\text { LCD } 8 X 2 \text { I2C }\end{array}$ & As Indicator \\
\hline 8 & FTDI 232 & $\begin{array}{l}\text { As Programming device from USB and Arduino } \\
\text { IDE to Arduino Pro mini }\end{array}$ \\
\hline 9 & $\begin{array}{l}\text { Regulator } 3.3 \text { Volt } \\
\text { to } 5 \text { Volt }\end{array}$ & $\begin{array}{l}\text { Change the voltage to HV (High Voltage 5Volt ) } \\
\text { or LV (Low Voltage } 3.3 \text { Volt) }\end{array}$ \\
\hline 10 & $\begin{array}{l}\text { Battery } 3.7 \text { Volt } \\
1000 \mathrm{mAh}\end{array}$ & Supply power to the sensor node \\
\hline
\end{tabular}

Actually the role is not important after the program upload process on Arduino pro mini, so it can be separated and the sensor node only uses the function of Arduino Pro Mini, but FTDI 232 is still useful besides being a bootloader, which is to supply 3.3 volts of power on ZigBee and 8X2 LCD. The role of supplying power to the node of this sensor is a 3.7 Volt 1000 mAh battery. The display of the prototype can be seen in Fig. 9. Accordingly, the position of the node or ZigBee end device is on it is necessary to see the color classification, there are three conditions, e.g. normal, represented in green (60-100 BPM), bradycardia (BPM $>44 \&<60$ BPM) or (BPM $>100 \&$ $<116)$ represented in yellow or a minor condition and Major or tachycardia (BPM $<44 \&>116$ BPM) are represented in red, therefore, classification can take place easily from pulse indications.

Fig. 10 is a sensor node that will be used in mesh networks using the Routing Protocol for Low Power and Lossy Network (RPL). This RPL is done using the simulation software with the IEEE 802.15.4 protocol.

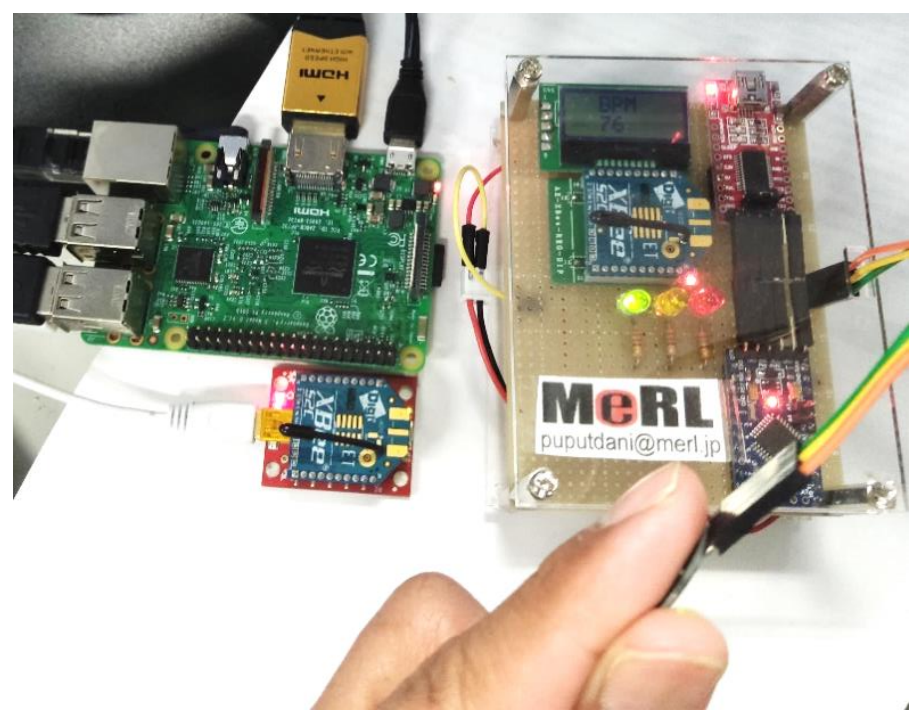

Fig. 9. Node Sensor.

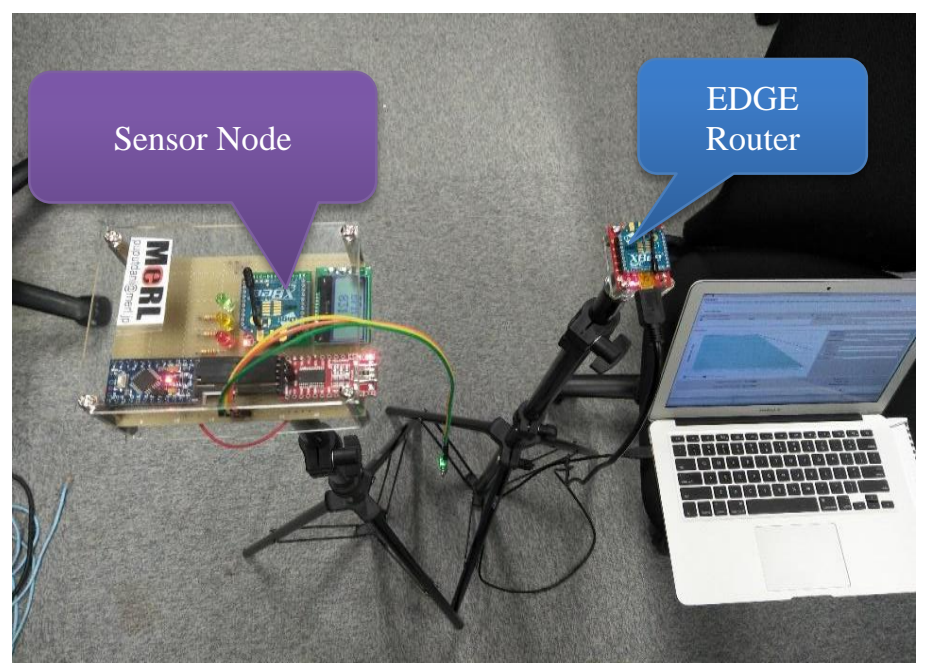

Fig. 10. ZigBee end Devices and ZigBee Coordinator Connectivity. 

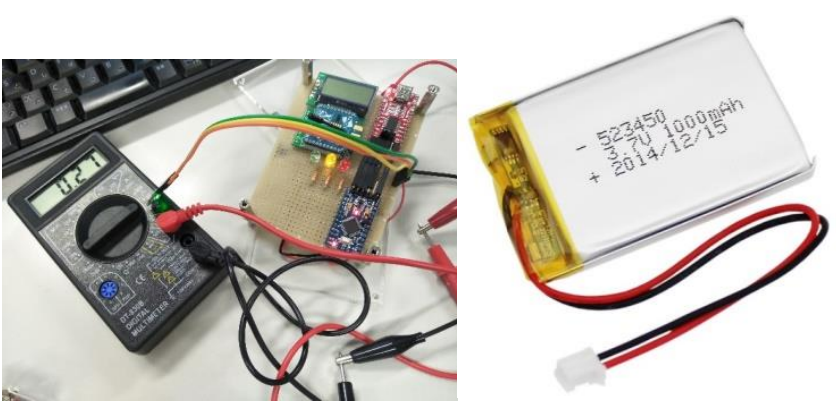

Fig. 11. Battery Lifetime Zigbee Pulse Sensor Node.

With the specifications of the 3.7 Volt Battery node 1000 $\mathrm{mAh}$, and load calculations e.g. XBee $=27 \mathrm{~mA}$ (TX Mode), LCD $8 \times 2=2 \mathrm{~mA}$, Arduino Pro mini $40 \mathrm{~mA}$, FTDI232 is 50 $\mathrm{mA}$ and Pulse $5 \mathrm{~mA}$, then total load is $124 \mathrm{~mA}$. With a 5 Volt Voltage, then the Power is Voltage $\mathrm{x}$ Current $=5 \mathrm{x} 0.124$ is 0.62 Watt. Then $\mathrm{I}=0.62 \mathrm{~W} / 3.7$ Volt $=0.167$ Ampere. Therefore, the power Consumption is $1000 \mathrm{mAH}$ or $1 \mathrm{AH} /$ $0.167 \mathrm{~A}=6$ hours, this measurement is shown in Fig. 11 .

\section{H. Equations of Received Signal Strength (dBm) and Path Loss (-dB) of IEEE 802.15.4 Zigbee}

Zigbee is a Radio Frequency (RF) device of $2.4 \mathrm{GHz}$ or $2400 \mathrm{MHz}$, so the wavelength $(\lambda)$ of zigbee is 0.125 meters, this value is obtained from $\lambda=\mathrm{c} / \mathrm{f}$, where $\mathrm{c}$ is the speed of light which is $3 \times 108$. Power receiver $(\mathrm{dB})$ will always weaken if distance (d) gets farther away equation (8), then the initial Power Receiver $(\operatorname{Pr} 0)$ when $\mathrm{d}=1 \mathrm{~m}$, can be seen in equation (1), equation (2).[3]

The first test parameter is RSS $(\mathrm{dBm})$ in the Free Space state, where the value of the exponent (n) of Free Space is 2. The value of the Receiver Signal Strength (RSS) results from the comparison of equation 10 and at the time of experiment or measurement in the field, while attenuation generated from equations 6,7 and 8 .

P0 $=$ Ptx. Gt. Gr. $(\lambda / 4 \pi)^{2}$

$\operatorname{Pr}=\frac{P 0}{d^{2}}$ (3).

A logarithmic form (decibel scale), can be seen in equation

$10 \log _{10} \mathrm{Pr}=10 \log _{10} \mathrm{P} 0-20 \log _{10} \mathrm{~d}$

$L$ Free Space can generally be calculated according to the equation (4).

L Free Space $=-(20 \log d+20 \log f-27,5)$

$\mathrm{C}$ is a constant value that is responsible for wireless attenuation in the Free Space condition which is worth 27.5. So that if distance (d) gets bigger, the value of L Free Space ($\mathrm{dB})$ will increase. So that the frequency of Zigbee is 2.45 $\mathrm{GHz}$, from equation 4 becomes equation (5).

L Free Space_2450 MHz $=-(20 \log \mathrm{d}+40.3)$

In Zigbee Datasheet Zigbee, attenuation in free space has a different value in equation (6).

L Free Space_2450 MHz $=-(33 \log \mathrm{d} / 8+58.5)$
In Fig. 15 shows the attenuation of Zigbee based on the value of $d$ which is getting bigger, so that the value of $L$ Free Space $(-\mathrm{dB})$ is getting bigger as well. In fact, on experiment 1 and experiment 2 Attenuation is affected by noise when transferring data on the Zigbee transmitter sensor to zigbee receiver sensor so that the data is not so smooth, but in outline decreases (-dB) or PathLoss value gets bigger. In Fig. 10 at a distance of $1 \mathrm{~m}$ Pathloss shows an average value of $-35 \mathrm{~dB}$ and at a distance of $50 \mathrm{~m}$ Pathloss average value of $-75.4 \mathrm{~dB}$. So that after the attenuation value $(-\mathrm{dB})$ has been found, the $\mathrm{Pr}$ value (Power Receiver $(\mathrm{dBm})$ can be determined by equation (7).

$\mathrm{Pr}=\mathrm{Pt}+\mathrm{Gr}+\mathrm{Gt}+\mathrm{L}$

Assuming the antenna gain for both transmitter and receiver is $0 \mathrm{~dB}(1 \mathrm{~mW})$ on the Zigbee specification, on free space propagation is formulated in equation (8). And for indoor propagation condition is shown in equation (9).

$\mathrm{Pr}=\mathrm{Pt}-(33 \log \mathrm{d} / 8+58.5)$

$\operatorname{Pr}=\mathrm{Pt}-(40 \log \mathrm{d} / 8+50.3)$

The specification of the Zigbee Power Transmitter datasheet shows the value of $0 \mathrm{dBm}$ for Low Power and +20 $\mathrm{dBm}$ for High Power Transmitters so that you can make a comparison chart. An example of sending data from the TX Zigbee pulse sensor Node to this RX Zigbee sensor node is shown in Fig. 12.

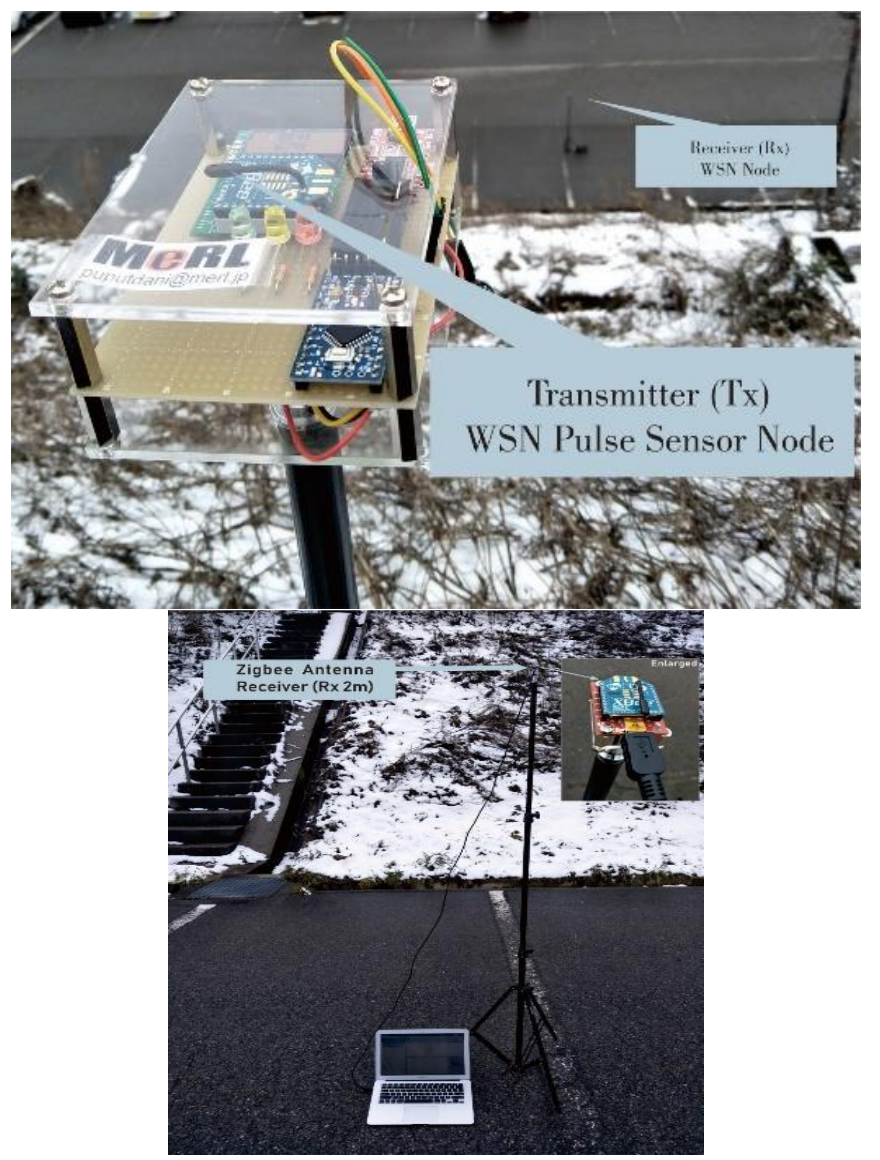

Fig. 12. Zigbee Transmitter and Receiver at Free Space Propagation. 


\section{DODAGs of Sensor Nodes Power Consumption Analyze}

In this research using Routing Protocol for Low Power and Lossy Network (RPL) analysis [7], so that the value of traffic sensor nodes can be generated in detail. During the simulation, the Sensor node or IEEE 802.15.4 parameter needs to be included in the simulated parameters, so the results can be compared with the experiment. In accordance with the Zigbee S2c specifications used in this research, the ZigBee $2.4 \mathrm{GHz}$ frequency, the ZigBee data rate is $250 \mathrm{kbps}$, and the transmission rate is $10-100$ meters on Line-of-sight area. in this simulation, the transmission rate $(\mathrm{Tx})$ range is 50 meters. Please note that when the sensor data reaches the sink, the analysis process continues at the next point. At the sensor node, when on the Tx range other sensor nodes are worth $100 \%$, meaning that they are included in the sink range or other sensor nodes that are the router, and the maximum distance is $\mathbf{5 0}$ meters. If the distance is $>\mathbf{5 0}$ meters, the sensor node is not detected and cannot be analyzed, consequently, the sensor node is required to enter in the range of 50 meters. Therefore, in the simulation of sensor nodes that enters the range of 50 meters indicated by a value of $100 \%$. The Power Consumption test parameters on the sensor node used include CPU, LPM, Radio Transmit (Tx) and Radio Listen (Rx).

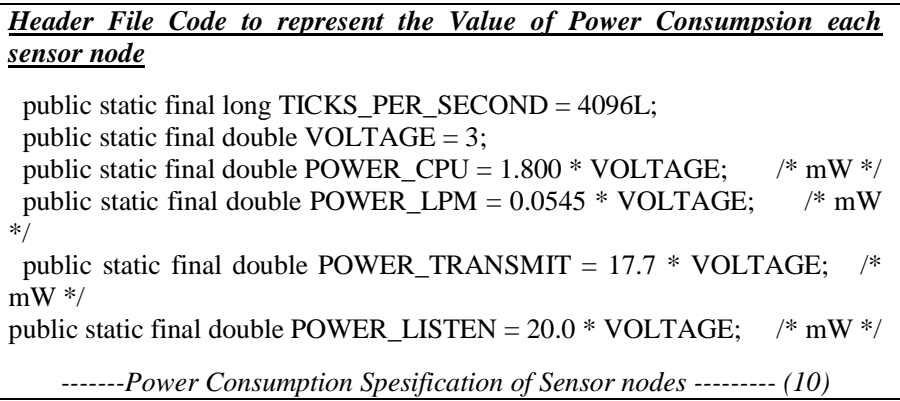

Equation the energy Consumption of Sensor node and relationship with time

*CPU energy:* $\mathrm{cp}=\mathrm{c} * 1.8 / \mathrm{tm}$

*LPM energy:* $\mathrm{lp}=1 * 0.0545 / \mathrm{tm}$

$*$ Transmit energy:* $1 \mathrm{t}=\mathrm{t} * 17.7 / \mathrm{tm}$

$*$ Listen energy:* $1 r=r * 20 / \mathrm{tm}$

$*$ Total energy: $* \mathrm{n}=\mathrm{cp}+\mathrm{lp}+\mathrm{lt}+\mathrm{lr}$

$\mathrm{tm}=\mathrm{c}+1$

where:

$* \mathrm{tm}^{*}$ is the total time

$*^{*}{ }^{*}$ is the time that the CPU was used.

* $1 *$ is the the time that the sensor was in Low Power Mode (LPM)

$* t *$ is the transmit time and

$*^{*} *$ is the Listen time

------Energy Consumption equation of Sensor nodes -------- (11)

In this research, the approach is carried out if there are many nodes that are interconnected with node specifications that have specifications in equation (10) and equation (11). With the DAGs and DODAGs method by setting the Sink number in the Clustering system, furthermore, how does the power consumptions affect and the analysis of changes in the position of nodes on the Routing Protocol for Low Power and Lossy Network (RPL) topology, the aim is to get a low Power Consumption on each node, furthermore, that the Average Average Power Consumption (mW) is obtained. Consequently, the test is done by setting the node position in
DODAG's Directed Acyclic Graph (DAG) and Destination (DAG).

In Fig. 13, the router node (node 3 ) has a different task, namely as a router node for all nodes joined in the DAGs topology. In this case, the Router node must have more Power to receive all data multiplexing from the data end nodes.

While in Fig. 14, divide the Router node into two nodes, namely Router node (2) and node (11) with the same number of nodes in Fig. 13 and Fig. 14, i.e. 10 sender nodes and 1 node coordinator (sink). This decreases the Power needed by the Router node to send sensor data to the coordinator node (sink). Fig. 17 and Fig. 18 show that Power Consumption is needed for each node, so that conclusions from 2 DODAG topology can be obtained which is the most appropriate to be applied when sending pulse sensor nodes in this research.

In Fig. 17 and Fig. 18, there is a significant difference between Topology DODAG 1 (Fig. 13) and DODAG 2 (Fig. 14), this difference is seen from the energy consumption required by the sensor node in communicating and sending data to other nodes (neighboard). In Fig. 17, the power consumption required by node 3 is much higher than the other 10 nodes, this is because node 3 is the directed node or the router node that sends all nodes to the sink or coordinator node. Consequently, Average Power consumption in Fig. 17 can be seen in Fig. 19, and the Average in the Power Consumption of 2 Router node can be seen in Fig. 20.

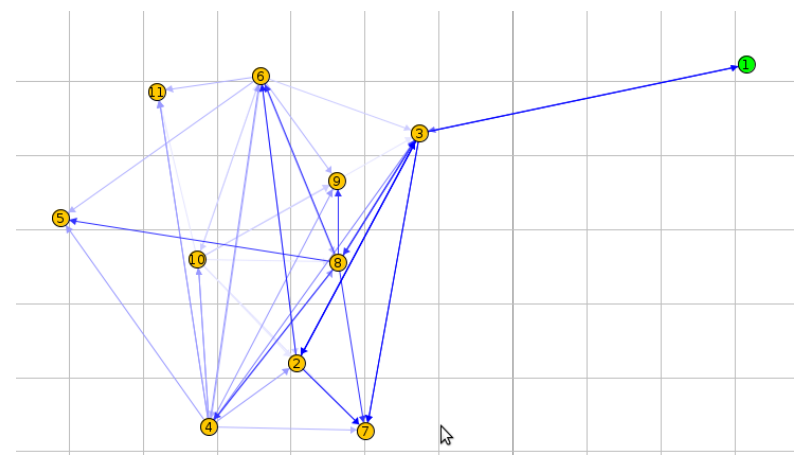

Fig. 13. Multi DAGs on DODAG.

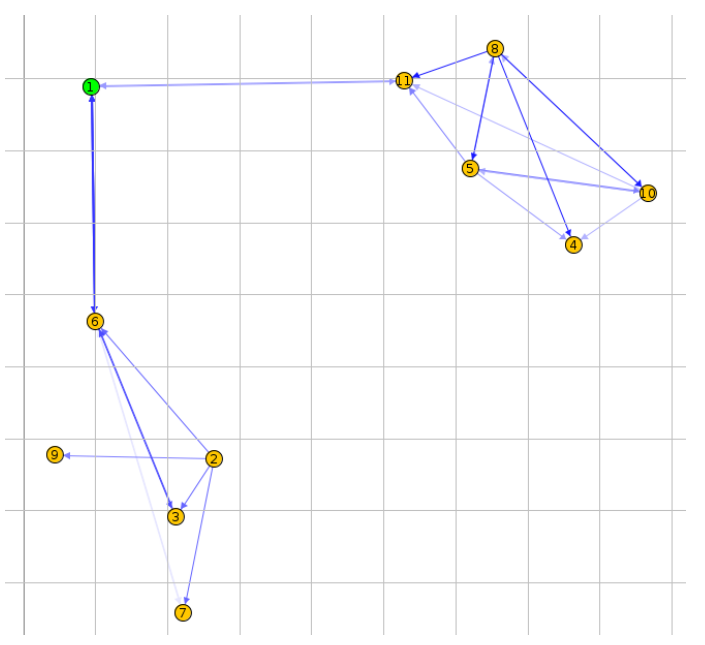

Fig. 14. DAGs with a Division Router. 


\section{RESUltS AND ANALYSIS}

Fig. 15 shows the PathLoss $(-\mathrm{dB})$ value for Free Space compared to Equation 4, 5 and 6 and 9 with - (40 log d / $8+$ 50.3 ) this is indoor position. The average of the 4 equations at a distance of $1 \mathrm{~m}$ is $-30.9 \mathrm{dBm}$ and at a distance of $50 \mathrm{~m}$ is $78.9 \mathrm{dBm}$.

Fig. 16 shows the value of the Power Receiver (dBm) based on the distance and value of the Power Transmitter if using the minimum Pr value $(0 \mathrm{dBm})$ and $\operatorname{Pr}$ value $(+20 \mathrm{dBm})$ at different distances. Fig. 16 is Comparing Graph from the Calculation value in equation (7) and the Power Receiver $(\mathrm{dBm})$ generated from the experiment in the field using the Digi X-CTU Zigbee software.

Fig. 19 and Fig. 20 are Average Power Consumption on 1 Router node and 2 Router nodes; sampling is for 10 minutes in both experiments on 1 and 2 Router nodes. From the experiment it can be concluded that using 1 Router Node will use Power Consumption greater than using 2 router nodes, with 1 router node that handles 10 sender nodes requires 1.35 $\mathrm{mW}$ Power Consumption while with 2 routers it can be reduced to an average $1 \mathrm{~mW}$ Power Consumption.

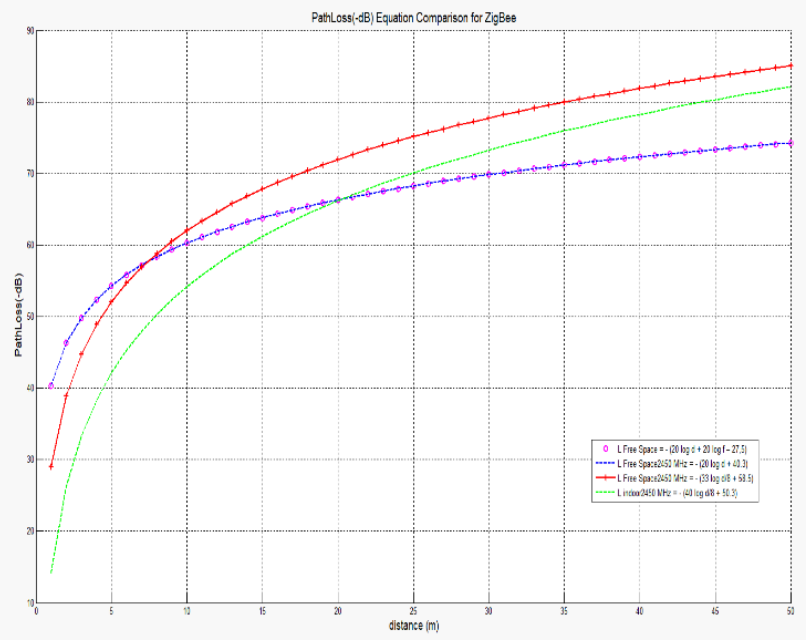

Fig. 15. ZigBee PathLoss $(-d B)$ on Free Space Area.

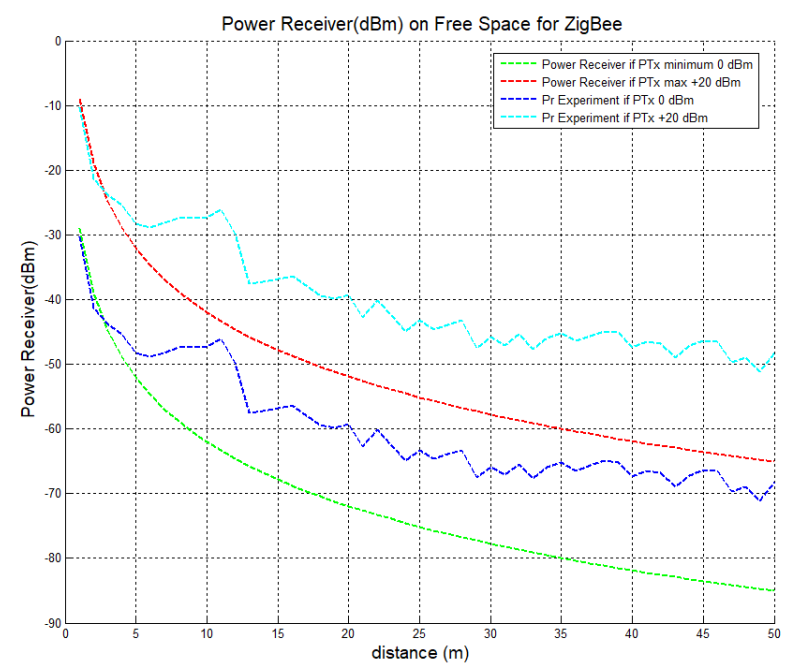

Fig. 16. Received Signal Strength of Zigbee $(d B m)$ on Free Space Area.

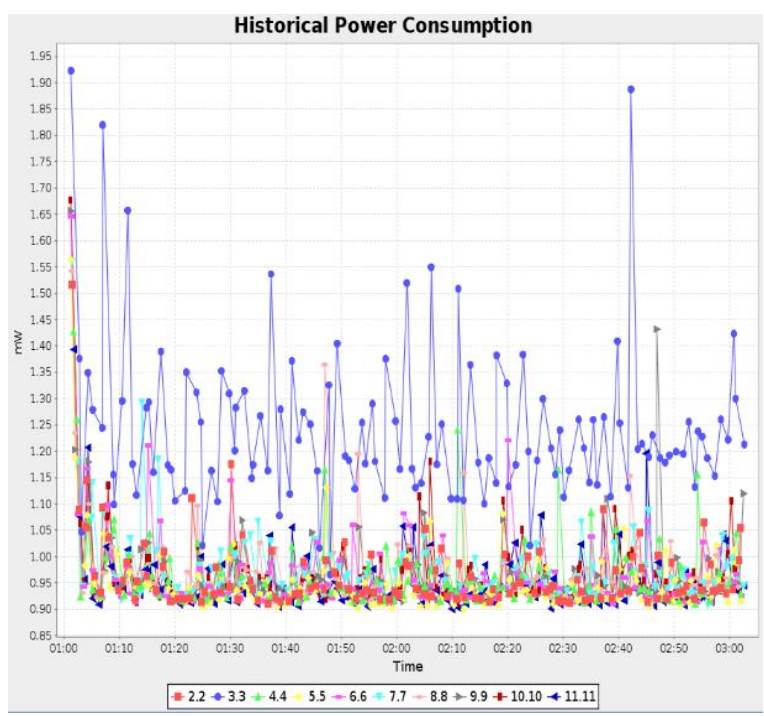

Fig. 17. Power Consumption of 1 Router Node.

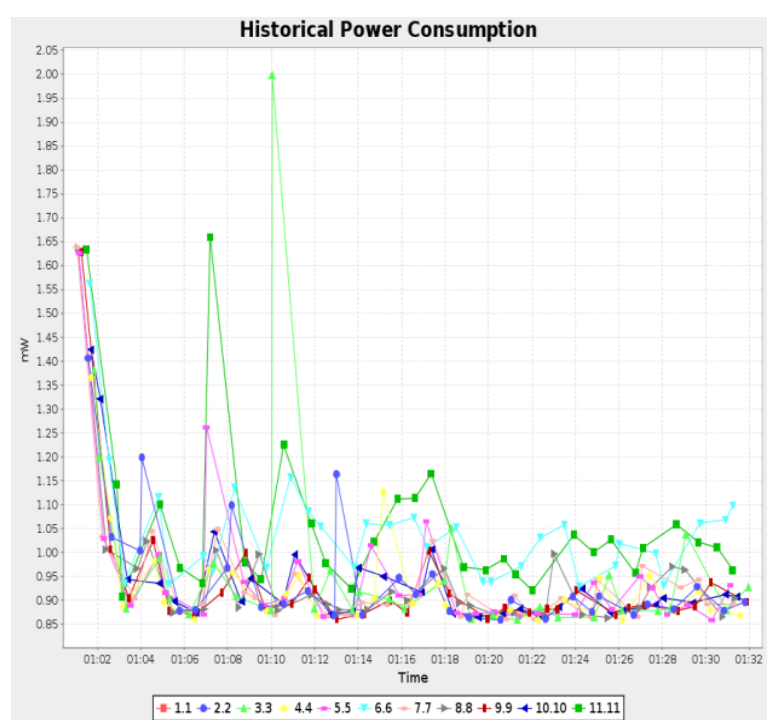

Fig. 18. Power Consumption of 2 Router Node.

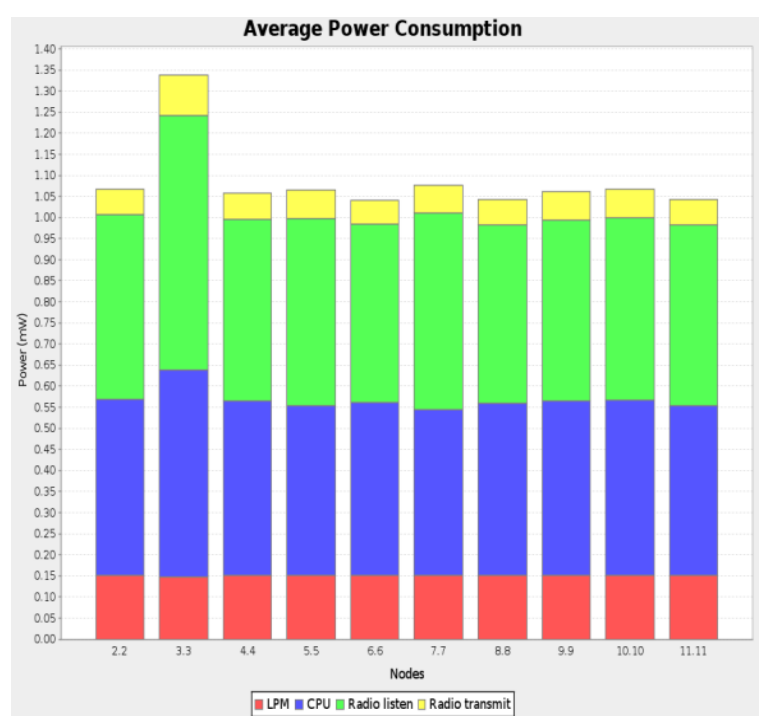

Fig. 19. Average Power Consumption of 1 Router Node. 


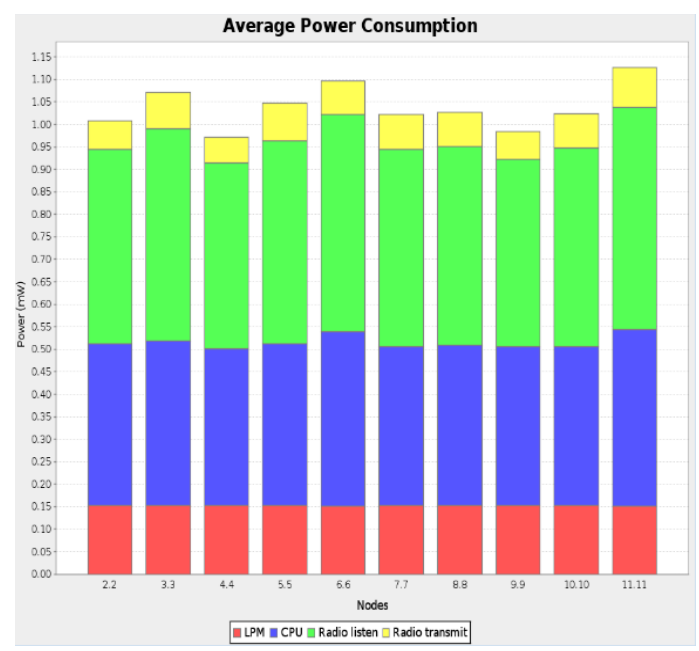

Fig. 20. Average Power Consumption of 2 Router Node.

In Fig. 21, Nodes 22, 23, 24 and 26 are Router nodes whose task is to send data from end devices to communicate with the coordinator node.

The conclusion that can be generated from analysis at Fig. 21 is that the more sensor nodes are in the position of end devices with a large number of hops that will not affect power consumption, however a power consumption is influenced by the number of end nodes handled by the node, for example, nodes 15 and 26 are nodes most in the DODAGs topology which has the highest number of nodes than the node handled by other Router nodes, see in Fig. 22. What is different is that node 15 has a number of hops 2 , meaning that its position is not as a router but as a node rank = 2, see in Fig. 23 .

In this section, the Pulse sensor data is in the Node Coordinator and is ready to be transferred to the MySQL database via the Python Language platform on the Internet Gateway. The internet gateway used is Raspberry Pi 3. Python language sends pulse sensor data to the MySQL database, then the data is sent from the MySQL database to HTML and JASON so that the pulse sensor data can be seen in real-time on the Internet, Fig. 24, according to the Internet of things architecture in Fig. 7.

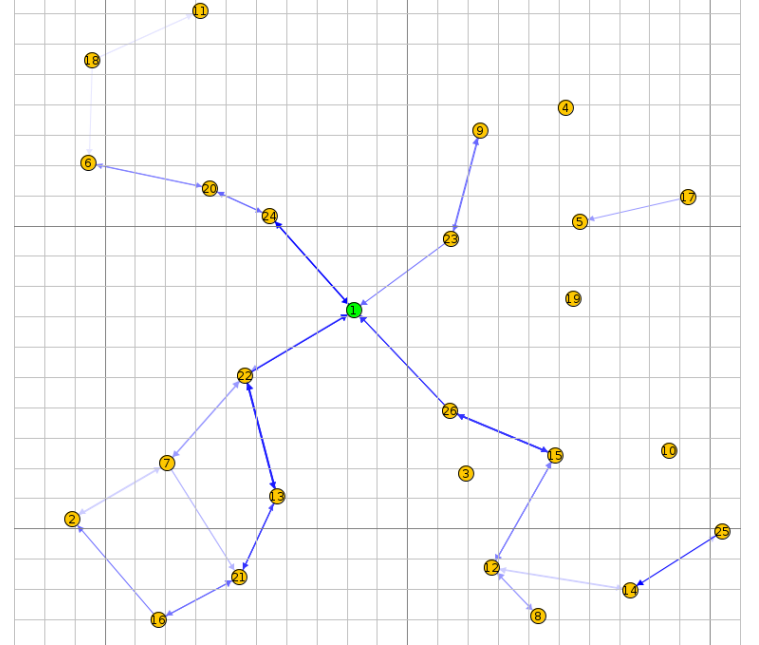

Fig. 21. DAGs with a 4 Division Router Node.

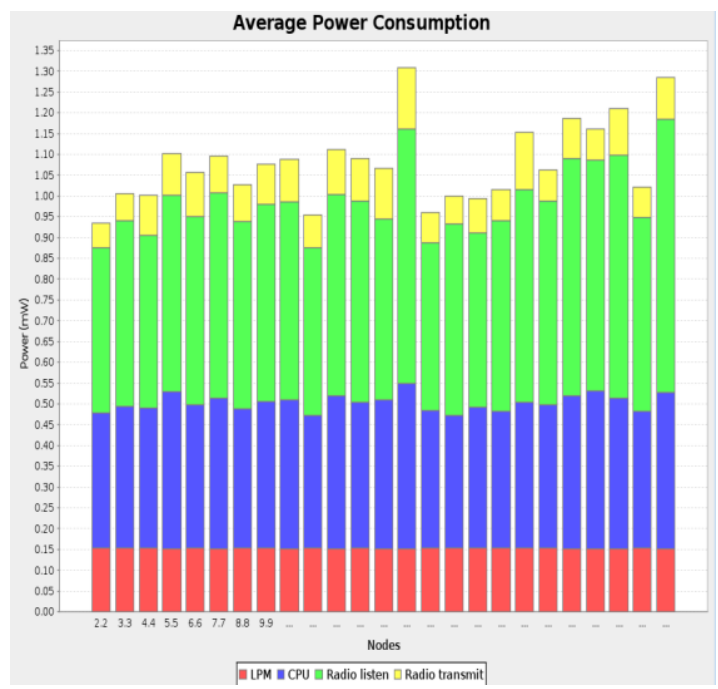

Fig. 22. Average Power Consumption of 26 Nodes.

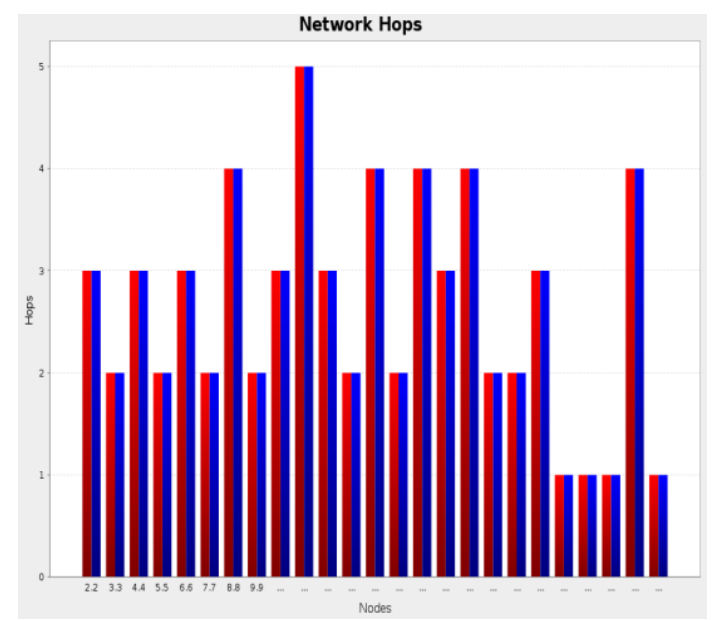

Fig. 23. Network Hops.

Display Chart of a Pulse (BPM) Real-Time Monitoring (MeRL)

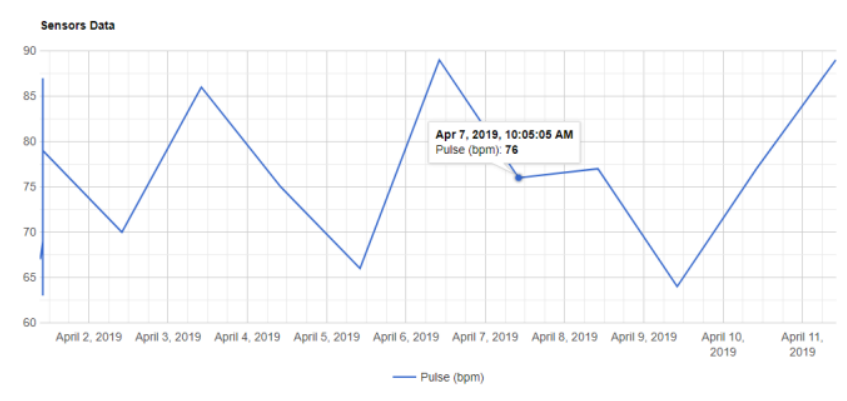

Fig. 24. Realtime Data Pulse (BPM) at Web Page HTML JASON.

\section{CONCLUSIONS}

The Quality of Services (QoS) from a Zigbee pulse sensor node can be seen from the parameters i.e. Pathloss (Attenuation (-dB)) and Power Receiver (Receiver Power Strength $(\mathrm{dBm})$ ), in this case, ZigBee PathLoss (-dB) on Free Space area will be represented by $1-50 \mathrm{~m}$ distances, consequently, the average of the 4 equations at a distance of 
$1 \mathrm{~m}$ is $-30.9 \mathrm{dBm}$ and at a distance of $50 \mathrm{~m}$ is $-78.9 \mathrm{dBm}$. Furthermore, the average Power Receiver $(\mathrm{dBm})$ on Free Space in $1-15 \mathrm{~m}$ distances for Zigbee is $-66,671 \mathrm{dBm}$. In the IEEE 802.15.4 routing sensor, the sensor node shows with 1 router node and the 10 sender nodes handle require $1.35 \mathrm{~mW}$ Power Consumption, this can be concluded that the more router nodes the sensor is Power Consumption will be lower, this is because the load given is not based on just one router node. Sending data from Sink node or EDGE node to Internet Gateway has been successful. Sensor Pulse Data is successfully stored in the MySQL database and displayed on the Web in real-time.

\section{DISCUSSION AND SUGGESTIONS}

This research will be applied to the Monitoring Health Patient Status, as long as there is internet connection, the Patient data e.g. Pulse will be very easy to obtain. In this research discuss about the performance of Zigbee devices at a distance of $50 \mathrm{~m}$ with the Coordinator node or receiver connected to the Raspberry $\mathrm{Pi} 3$ as an internet gateway. Analysis of future research will also discuss the comparison of topology in ZigBee in the free space area so that the data pulse will be analyzed based on details of QoS based on the ZigBee topology and its impact on the ZigBee coordinator node. Furthermore, the data pulse for the ZigBee coordinator node will be sent via Raspberry Pi 3 as an internet gateway using Python Programming. This research still has to be developed with the analysis of the Internet of Things Protocol and Security Methods of the Internet of Things. Comparison of 6LOWPANs, Zigbee and LoRAWAN can be done in the next research. Development of the Routing Algorithm needs to be done to obtain a low Energy Consumption value on the Sensor node.

\section{ACKNOWLEDGMENT}

Thanks to Micro Electronics Research Laboratory (MeRL) and Professor Kitagawa as a MeRL Leader for guiding, providing tools and laboratories Analyzers, this research paper can be resolved properly, hopefully this paper can be used as a reference for the world of health, specifically the development of Wireless Sensor Networks technology for monitoring health status based on the Internet of Things.

\section{REFERENCES}

[1] Muhammad Niswar, Amil Ahmad Ilham, Elyas Palantei, Rhiza S Sadjad, Andani Ahmad, Ansar Suyuti, Indrabayu, Zaenab Muslimin, Tadjuddin Waris, Puput Dani Prasetyo Adi, Performance evaluation of ZigBee-based wireless sensor network for monitoring patients' pulse status, 2013 International Conference on Information Technology and Electrical Engineering (ICITEE) DOI: doi/10.1109/ICITEED.2013.6676255

[2] Jinze Du, Jean-François Diouris and Yide Wang, A RSSI-based parameter tracking strategy for constrained position localization, Du et al. EURASIP Journal on Advances in Signal Processing (2017) 2017:77, DOI 10.1186/s13634-017-0512-x

[3] Jiyan Huang, Peng Liu, Wei Lin and Guan Gui, RSS-Based Method for Sensor Localization with Unknown Transmit Power and Uncertainty in Path Loss Exponent, Sensors 2016, 16, 1452; doi:10.3390/s16091452

[4] Jungang Zheng, Yue Liu, Xufeng Fan and Feng Li, The Study of RSSI in Wireless Sensor Networks, Advances in Intelligent Systems Research, volume 133, 2nd International Conference on Artificial Intelligence and Industrial Engineering (AIIE2016) Copyright (C) 2016, the Authors. Published by Atlantis Press.
[5] P. K. Dutta, O. P. Mishra, and M. K. Naskar, Analysis of dynamic path loss based on the RSSI model for rupture location analysis in underground wireless sensor networks and its implications for Earthquake Early Warning System (EEWS), International Journal of Automation and Smart Technology (AUSMT), DOI: 10.5875/ausmt.v5i3.858

[6] Pooyan Abouzar, David G. Michelson, and Maziyar Hamdi, RSSI-Based Distributed Self-Localization for Wireless Sensor Networks used in Precision Agriculture, arXiv:1509.02400v1 [cs.DC] 21 Aug 2015, https://arxiv.org/pdf/1509.02400.pdf

[7] Puput Dani Prasetyo Adi and Akio Kitagawa, "Performance Evaluation WPAN of RN-42 Bluetooth based (802.15.1) for Sending the MultiSensor LM35 Data Temperature and RaspBerry Pi 3 Model B for the Database and Internet Gateway" International Journal of Advanced Computer Science and Applications (IJACSA), 9(12), 2018. DOI: dx.doi/10.14569/IJACSA.2018.091285

[8] Pushan Dutta, O.P.Mishra, M.K.Naskar, Analysis of dynamic path loss based on the RSSI model for rupture location analysis in underground wireless sensor networks and its implications for Earthquake Early Warning System (EEWS), September 2015 International Journal of Automation and Smart Technology 5(3), DOI: 10.5875/ausmt.v5i3.858

[9] Ranjan Kumar Mahapatra, N. S. V. Shet, Localization Based on RSSI Exploiting Gaussian and Averaging Filter in Wireless Sensor Network, Arabian Journal for Science and Engineering, August 2018, Volume 43, Issue 8, pp 4145-4159, DOI: doi/10.1007/s13369-017-2826-2

[10] Yu Xiaoqing, Zhang Zenglin, Han Wenting, Experiment Measurements of RSSI for Wireless Underground Sensor Network in Soil, IAENG International Journal of Computer Science, 45:2, IJCS_45_2_02, Advance online publication: 28 May 2018

[11] Puput Dani Prasetyo Adi and Rahman Arifuddin, Design of Tsunami Detector Based Sort Message Service Using Arduino and SIM900A to GSM/GPRS Module, JEEMECS (Journal of Electrical Engineering, Mechatronic and Computer Science) Volume 1, No.1. 2018, DOI: doi/10.26905/jeemecs.v1i1.1982

[12] Umair Mujtaba Qureshi, Faisal Karim Shaikh, Zuneera Aziz, Syed M. Zafi S. Shah, Adil A. Sheikh, Emad Felemban and Saad Bin Qaisar, RF Path and Absorption Loss Estimation for Underwater Wireless Sensor Networks in Different Water Environments, Sensors 2016, 16(6), 890; https://doi.org/10.3390/s16060890

[13] Daihua Wang, Linli Song, Xiangshan Kong, and Zhijie Zhang, NearGround Path Loss Measurements and Modeling for Wireless Sensor Networks at $2.4 \mathrm{GHz}$, Hindawi Publishing Corporation International Journal of Distributed Sensor Networks Volume 2012, Article ID 969712, 10 pages doi:10.1155/2012/969712

[14] Hana Mujlid, Ivica Kostanic, Propagation Path Loss Measurements for Wireless Sensor Networks in Sand and Dust Storms, Frontiers in Sensors (FS) Volume 4, 2016, DOI: doi/10.14355/fs.2016.04.004 www.seipub.org/fs

[15] Hristos T. Anastassiu, Stavros Vougioukas, Theodoros Fronimos, Christian Regen, Loukas Petrou, Manuela Zude 4 and Jana Käthner, A Computational Model for Path Loss in Wireless Sensor Networks in Orchard Environments, Sensors 2014, 14, 5118-5135; doi: $10.3390 / \mathrm{s} 140305118$

[16] J. Miranda, R. Abrishambaf, T. Gomes, P. Gonçalves, J. Cabral, A. Tavares and J. Monteiro, Path Loss Exponent Analysis in Wireless Sensor Networks: $\quad$ Experimental https://www.researchgate.net/publication/256733582, Conference Paper July 2013, DOI: 10.1109/INDIN.2013.6622857

[17] Michael Cheffena and Marshed Mohamed, Empirical Path Loss Models for Wireless Sensor Network Deployment in Snowy Environments, IEEE Antennas and Wireless Propagation Letter (Volume:16), 11 September 2017, DOI: 10.1109/LAWP.2017.2751079

[18] Naseer Sabri, S A Aljunid, M S Salim, R Kamaruddin, R B Ahmad, M F Malek, Path Loss Analysis of WSN Wave Propagation in Vegetation, Journal of Physics: Conference Series 423 (2013) 012063, doi:10.1088/1742-6596/423/1/012063, ScieTech 2013, IOP Publishing.

[19] Sinant Kurt and Bulent Tavli, Path Loss Modeling for Wireless Sensor Network : Review of Models and Comparative Evaluations, IEEE Antennas and Propagation Magazines, July 2016, DOI:10.1109/MAP.2016.2630035 
[20] Tajudeen O. Olasupo, Carlos E. Otero, Kehinde O. Olasupo, Ivica Kostanic, Empirical Path Loss Models for Wireless Sensor Network Deployments in Short and Tall Natural Grass Environments, IEEE Transactions on Antennas \& Propagation, 2016, Manuscript ID is AP1512-1931.R2, DOI 10.1109/TAP.2016.2583507, IEEE Transactions on Antennas and Propagation

[21] Xiaoqing Yu, Wenting Han, Zenglin Zhang, Path Loss Estimation for Wireless Underground Sensor Network in Agricultural Application, Agric Res (2017) 6: 97. DOI: doi/10.1007/s40003-016-0239-1

[22] Zhenran Gao, Weijing Li, Yan Zhu, Yongchao Tian, Fangrong Pang, Weixing Cao, and Jun Ni, Wireless Channel Propagation Characteristics and Modeling of Research in Rice Field Sensor Networks,Sensors 2018, 18, 3116; doi/10.3390/s18093116

[23] Jose Vera-Pérez, David Todolí-Ferrandis, Salvador Santonja-Climent, Javier Silvestre-Blanes, and Víctor Sempere-Payá, "A Joining Procedure and Synchronization for TSCH-RPL Wireless Sensor Networks", Sensors 2018, 18, 3556; doi:10.3390/s18103556
[24] Puput Dani Prasetyo Adi, Analisis kinerja jaringan sensor nirkabel untuk monitoring denyut nadi pasien, April 2018, DOI: 10.13140/RG.2.2.29145.83040

[25] Pradana, H., \& Adi, P. (2018). Monitoring Detak Jantung Dan Sistem Implementasi Telemetri Pada Pelaksanaan Lari. SinarFe7, 1(2), 552556. Retrieved from http://ejournal.fortei7.org/index.php /SinarFe7 /article/view/187

[26] Widya Cahyadi, Muhammad Arief Wahyudi, dan Catur Suko Sarwono, "Analisis Perbandingan Konsumsi Energi dan Masa Hidup Jaringan pada Protokol LEACH, HEED, dan PEGASIS di Wireless Sensor Network", Jurnal Rekayasa Elektrika, VOLUME 14 NOMOR 2, AGUSTUS 2018

[27] Muhammad Ateeq, Farruh Ishmanov, Muhammad Khalil Afzal, and Muhammad Naeem" Multi-Parametric Analysis of Reliability and Energy Consumption in IoT: A Deep Learning Approach", Sensors 2019, 19, 309; doi:10.3390/s19020309 